\title{
INJURY TO PRESENTATION DELAYS AMONG MUSCULOSKELETAL TRAUMA PATIENTS IN UGANDA
}

J.W.M. Kigera, MBChB, MMed, Resident, Department of Orthopaedics, Makerere University, Kampala, Uganda, Orthopaedic Surgeon,Kikuyu Orthopaedic Rehabilation Center, PCEA Kikuyu Hospital Kenya and E.K. Naddumba, MBChB, MMed, FCS (ECSA) Senior Consultant Surgeon, Department of Orthopaedics, Mulago Hospital, Kampala, Uganda

Correspondence to: Dr. J.W.M. Kigera, Orthopaedic Rehabilitation Centre, PCEA Kikuyu Hospital, Kenya.

Email: jameskigera@yahoo.co.uk

\section{ABSTRACT}

Background: Injuries are a common cause of morbidity and mortality in the developing world with road traffic crashes being especially prevalent. Kampala has no organised pre hospital rescue service and this causes delays in presentation to hospital with possible adverse outcomes.

Objective: The study sought determine the time it took patients to arrive in the hospitals and document the various factors associated with delays in presentation to hospital.

Design: Prospective study.

Setting: Accident and Emergency Department, Mulago Hospital , Kampala, Uganda

Methods: All musculoskeletal injury patients presenting to Mulago Hospital were prospectively enrolled and details of the injury including times of injury and presentation to hospital recorded. Data analysis was done and the Man-Whitney $U$ test and chi square tests used for statistical tests.

Results: The median delay in presentation was 3.5 hours. Factors associated with delayed presentation included being managed as an outpatient, having an upper limb injury, being less than 18 years of age and being involved in a road traffic crashes RTC.

Conclusion: The delay in presenting to hospital was comparable to other sites in East Africa though significantly longer than in the developed world. The prevalence of traditional bone setters and the lack of an organised pre hospital rescue service may be influencing the delay in presentation to hospital.

\section{INTRODUCTION}

Injuries are common cause of morbidity and mortality in the developing world(1). Road traffic crashes are causative in a majority of trauma patients seen in hospitals in Kampala (2-4). These crashes result in musculoskeletal injuries in a majority of cases (3, 5). In Kampala, there are no pre hospital services and most patients arrive by private transport after sustaining injuries while others are brought in by the police (6). Majority of injuries in Kampala are also known to occur in the streets and at home and pre hospital services would aid in saving lives, reducing further injury and speeding up the transportation to hospital (2).

Delay in presentation adversely affects outcome and survivorship in trauma patients. It has been shown that patients with hip fractures who present within 6 hours are in a better physiological state and they have better outcomes (7).
We postulated that the delay in presentation to hospital would be longer than in regions that have organised pre hospital services. We also sought to document the factors that are associated with this delay in presentation to hospital including demographic and injury factors. Knowing the adverse effect on outcome of patients that delay in presentation and management has, documenting these delays will form a basis for lobbying for the introduction of a pre hospital care service in Kampala and the whole country. Determining the risk factors for such delays would also help in determining which patients are at a higher risk for poorer outcomes.

\section{MATERIALS AND METHODS}

This prospective study included all musculoskeletal trauma patients who presented between August and December 2009 to the Accident and Emergency 
Department of Mulago Hospital. All patients seen as outpatients or inpatients in the Accident and Emergency Department with musculoskeletal injuries were included. Patients with fractures and dislocations of the extremities and spine injuries were included. We excluded patients with isolated trauma to the head, chest and abdomen. We also excluded patients with burns and pathological fractures and soft tissue injuries to the extremities. Patient demographic data were obtained by interviewing the patients while injury details were obtained from the case notes of the admitting doctor and review notes of the resident on call. The times of injury and presentation to hospital were obtained from history and were estimated to the nearest half hour. The upper limb was considered to include the scapula and clavicle while the lower limb included the pelvis and acetabulum. The data was collected by means of a questionnaire designed for that purpose by one author (JWMK). Patients for whom there was incomplete data were excluded from the analysis. The patients' injuries were not scored using any of the validated scoring systems. The study was conducted in Mulago Hospital, the National Referral Hospital in Uganda whose characteristics have been previously described (3). The study was approved by the institution review board. Data was entered in MS Excel and later exported into SPSS for windows version 11.5 (SPSS Inc., Chicago, Illinois). Statistical analysis was done using the Man-Whitney $\mathrm{U}$ test .

\section{RESULTS}

Out of the 537 patients seen in that period 441 (82.1\%) had valid data for both time of injury and arrival to hospital. The median delay in presentation to hospital was 3.5 hours (Table 1 ).

Table 1

Time delay of the patients

\begin{tabular}{lll}
\hline Category & No. & $\%$ \\
\hline Within 1 hour & 98 & 22.2 \\
1- 6 Hours & 192 & 43.5 \\
6-12 hours & 30 & 6.8 \\
12- 24 hours & 41 & 9.3 \\
1 day to 1 week & 71 & 16.1 \\
More than 1 week & 9 & 2.0 \\
\hline
\end{tabular}

The patients managed as outpatients comprised $35.3 \%$ of all patients with the rest $(64.7 \%)$ requiring admission for their injuries. The median delay in presentation among patients managed as outpatients was 12.5 hours while that of inpatients was 2.5 hours and this difference was statistically significant $(p<0.001)$. When the limb injuries were considered, the median delay in upper limb injuries was 5.5 hours while in lower limb injuries the delay was 2.5 hours. This difference was statistically significant $(p<0.001)$. Patients aged less than 18 years had a median delay of 5 hours compared to a delay of 2.5 hours in patients aged 18 years and above. This difference was statistically significant $(p<0.001)$.

There were differences noted in the delay and the mechanism of injury. Patients who were involved in road traffic crashes were more likely to have a shorter delay than those injured in all other mechanisms $(p<0.001)$. The mean delay in males was 3 hours while that in females was 4 hours and the difference was not statistically significant $(p=0.04)$. Majority of the patients had isolated injuries with only $9.8 \%$ of the patients having injuries in multiple sites. Patients with multiple injuries had a median delay in presentation of 2 hours compared to 4 hours among patients with isolated injuries. This difference was not statistically significant $(p=0.06)$.

Among patients aged 18 years and above, the education level did not influence the delay in presentation. Those who had no education or up to primary level education had a median delay of 3.5 hours while those who had attained a level of secondary school and above had a median delay of 2 hours. This difference was not statistically significant $(p=0.381)$.

Table 2

Factors associated with delayed presentation

\begin{tabular}{|c|c|c|}
\hline $\begin{array}{l}\text { Predictor } \\
\text { variable }\end{array}$ & Categories & $P$ value \\
\hline Age & $\begin{array}{l}<18 \text { years } \\
\text { vs. }>18 \text { years }\end{array}$ & $<0.001$ \\
\hline $\begin{array}{l}\text { Admission } \\
\text { status }\end{array}$ & $\begin{array}{l}\text { Outpatient } \\
\text { vs. inpatient }\end{array}$ & $<0.001$ \\
\hline Limb injuries & $\begin{array}{l}\text { Upper limb } \\
\text { vs. lower limb }\end{array}$ & $<0.001$ \\
\hline $\begin{array}{l}\text { Mechanism } \\
\text { of injury }\end{array}$ & $\begin{array}{l}\text { RTA vs. } \\
\text { all other }\end{array}$ & $<0.001$ \\
\hline Sex & Male vs. female & 0.04 \\
\hline Injuries & Isolated vs. multiple & 0.06 \\
\hline Education level & $\begin{array}{l}\text { None or primary } \\
\text { vs. secondary and above }\end{array}$ & 0.381 \\
\hline
\end{tabular}




\section{DISCUSSION}

Our average delay in presentation was 18 hours (median 3.5 hours) with only $22.2 \%$ of the patients arriving within the 'golden hour of trauma'. A study of all trauma patients done in Mulago and Kawolo Hospitals in Uganda found a mean delay of about 22.4 hours which is comparable to our results (8). Our results are however in variance to other studies found in literature. Otieno et al (4) in Kenya found a mean delay of 9 hours with only $18 \%$ presenting within 1 hour. Their study included all patients who had trauma but he excluded outpatient cases. When we considered inpatients only, our results are comparable with mean delay of 9.47 hours. Our findings are also in variance to another study done in Kampala(2) where $66 \%$ of the patients arrived in hospital within an hour of injury. However in this study majority $(97 \%)$ of the patients had minor injuries when compared to the patients in our study (2). A study on patients who had sustained hip fractures in Lithuania found a mean time of 50 hours (median 6 hours). This was done in a city where the estimated time from notifying health workers to arrival in hospital is about 2 hours (7). In the same study it was noted that the delay in presentation was more important than the delay to surgical intervention in determining outcome.

Sabharwal et al(9) study showed that on average children presented to a University Hospital emergency departments 2.5 days after injury. These patients had been seen in other Emergency Departments at an average of 0.5 days after injury.

The lack of an organised pre hospital care system in the city and indeed the whole country is hampering the delivery of health services. Majority of patients who present to hospital after 24 hours are injured outside the city and their transport may be inadequate further aggravating their injuries (2). Patients who were managed as outpatients presented late to hospital when compared to inpatients. It is plausible to conclude that patients who were eventually managed as outpatients had less severe injuries and did not urgently seek medical attention. It is also possible that these are the patients who are more likely to seek treatment outside the formal health system. Patients involved in road traffic crashes are usually, though not always more severely injured when compared to patients who fell or were injured in sports. Though this is not a formal severity scoring system it gives an indicator of the severity of the injuries. This may explain the differences in delay in presentation to hospital.

Our study did not investigate the individual reasons for delayed presentation. It is however known that many patients in Kampala and indeed in Uganda seek services from traditional healers before seeking formal medical care. Other authors(10) in Africa have also noted that only a fraction of injured patients are seen in the formal health facilities with patients in urban centres more likely to use the formal health facilities when compared with their rural counterparts (10).

Limitations in our study include its inability to differentiate between referral cases and cases presenting to the Accident and Emergency Department for the first time and the failure to score the severity of the injuries sustained. This would have been possible had there been a trauma registry at the hospital. It is however known that in Kenya the number of patients who are seen in small health facilities prior to referral to a main hospital is $52 \%$ (4). The establishment and maintenance of a trauma registry is difficult without having dedicated personnel for this(8). Previous studies, however have shown that about $20 \%$ of trauma patients in Kampala sustain injuries outside the district and are likely to have been seen in other health facilities(2). This study also did not look at the outcomes of the injured patients.

\section{CONCLUSION}

The delay in presentation to hospital in musculoskeletal trauma patients may be contributing to poorer outcomes. This problem needs to be addressed in a multi pronged approach. Establishment of a formal pre hospital trauma service will greatly reduce the delays that are currently experienced.

\section{REFERENCES}

1. Krug, E. Injury: a leading cause of global burden of disease. WHO; 1999.

2. Kobusingye, O.C., Guwatudde, D., Owor, G. and Lett, R.R. Citywide trauma experience in Kampala, Uganda: a call for intervention. Injury Prevention. 2002;8:133-136.

3. Kigera, J.W. M. and Naddumba, E. K. Patterns of injuries after Road Traffic Crashes involving Bodabodas. Annals Afr. Surg. 2010; 5:12-15.

4. Otieno, T., Woodfield, J.C., Bird, P. and Hill, A.G. Trauma in rural Kenya. Injury. 2004; 35(12):1228-1233.

5. Galukande, M., Jombwe, J., Fualal, J. and Gakwaya, A. Boda-boda injuries is a health problem and a burden 
of disease in Uganda: a tertiary hospital survey. East Central Afr. J. Surg. 2009; 14 (2 ):33-37.

6. Andrews, N., Kobusingye, O.C. and Lett, R. Road traffic accident injuries in Kampala. East Afr. Med. J. 1999; 76:189-194.

7. Vertelis, A., Robertsson, O., Tarasevicius, S. and Wingstrand, H. Delayed hospitalization increases mortality in displaced femoral neck fracture patients. Acta Orthop. 2009; 80(6):683-686.

8. Kobusingye, O.C. and Lett, R. Hospital based trauma registries in Uganda. J. Trauma. 2000; 48: 498-502.
9. Sabharwal, S., Zhao, C., McClemens, E. and Kaufmann, A. Pediatric orthopaedic patients presenting to a university emergency department after visiting another emergency department demographics and health insurance status. J. Pediat. Orthoped. 2007;27:690-694.

10. Mock, C.N., Nii-Amon-Kotei, D. and Maier, R.V. Low utilization of formal medical services by injured persons in a developing nation: health service data underestimate the importance of trauma. J. Trauma. 1997; 42: 504-513. 